\title{
HAMILTON SEQUENCES FOR EXTREMAL QUASICONFORMAL MAPPINGS OF DOUBLY-CONNECTED DOMAINS
}

\author{
GUOWU YAO
}

(Received 14 September 2012; accepted 21 December 2012; first published online 22 March 2013)

\begin{abstract}
Let $T(S)$ be the Teichmüller space of a hyperbolic Riemann surface $S$. Suppose that $\mu$ is an extremal Beltrami differential at a given point $\tau$ of $T(S)$ and $\left\{\phi_{n}\right\}$ is a Hamilton sequence for $\mu$. It is an open problem whether the sequence $\left\{\phi_{n}\right\}$ is always a Hamilton sequence for all extremal differentials in $\tau$. $\mathrm{S}$. Wu ['Hamilton sequences for extremal quasiconformal mappings of the unit disk', Sci. China Ser. A 42 (1999), 1033-1042] gave a positive answer to this problem in the case where $S$ is the unit disc. In this paper, we show that it is also true when $S$ is a doubly-connected domain.
\end{abstract}

2010 Mathematics subject classification: primary 30C75; secondary 30C62.

Keywords and phrases: Teichmüller space, Hamilton sequence.

\section{Introduction}

Let $S$ be a Riemann surface whose universal covering surface is the unit disc $\{z \in \mathbb{C}$ : $|z|<1\}$, and let $S$ be represented by a Fuchsian group $\Gamma$ acting on $\Delta$ as $S=\Delta / \Gamma$. Let $Q C(S)$ be the space of all quasiconformal mappings $f$ from $R$ to a variable Riemann surface $f(S)$. The Teichmüller space $T(S)$ is the space of these mappings factored by an equivalence relation. A quasiconformal mapping $f$ in $Q C(S)$ can be lifted to a quasiconformal mapping $\widetilde{f}$ from $\Delta$ onto itself. Two mappings, $f$ and $g$, are equivalent (and therefore their Beltrami differentials are called equivalent) if there exist lifts $\widetilde{f}, \widetilde{g}$ of $f, g$ such that $\widetilde{f}$ agrees with $\widetilde{g}$ on $\partial \Delta$. Let $[f]$ or $[\mu]$ denote the equivalence class of a quasiconformal mapping $f$ in $Q C(S)$, where $\mu$ is the Beltrami differential of $f$. Since the Beltrami differential $\mu$ uniquely determines the mapping $f$ up to postcomposition by a conformal mapping, the Teichmüller space $T(S)$ may be represented as the space of equivalence classes of Beltrami differentials $\mu$ in the unit ball $M(S)$ of the space $L^{\infty}(S)$. The equivalence class of the Beltrami differential zero is the basepoint of $T(S)$.

The author was supported by the National Natural Science Foundation of China (Grant No. 11271216).

(c) 2013 Australian Mathematical Publishing Association Inc. 0004-9727/2013 \$16.00 
Given $f \in Q C(S)$, let $\mu \in$ be the Beltrami differential of $f$. We define

$$
k_{0}([\mu])=\inf \left\{\|v\|_{\infty}: v \in[\mu]\right\}
$$

A quasiconformal mapping $f$ of $S$ onto $f(S)$ is said to be extremal in its class $[f]$ if its Beltrami differential $\mu$ is extremal in $[\mu]$, that is, $\|\mu\|_{\infty}=k_{0}([\mu])$. Note that $[\mu]$ may contain more than one extremal element.

Let $\mathcal{A}(\Gamma)$ denote the Banach space of holomorphic quadratic differentials $\phi$ on $S=\Delta / \Gamma$ with $L^{1}$-norm

$$
\|\phi\|_{S}=\iint_{S}|\phi(z)| d x d y<\infty .
$$

Let $\mathcal{A}_{1}(\Gamma)$ denote the unit sphere of $\mathcal{A}(\Gamma)$. In particular, we denote by $\mathcal{A}(1)$ the the Banach space of integrable holomorphic quadratic differentials on $\Delta$.

The following theorem due to Hamilton, Kruškál, Reich and Strebel is a characterisation of extremal quasiconformal mappings (see [2]).

Theorem A. A quasiconformal mapping $f$ of $S$ is extremal if and only if its Beltrami differential $\mu$ has a so-called Hamilton sequence $\left\{\phi_{n}: \phi_{n} \in \mathcal{A}_{1}(\Gamma)\right\}$ such that

$$
\lim _{n \rightarrow \infty}\left|\iint_{S} \mu(z) \phi_{n}(z) d x d y\right|=\|\mu\|_{\infty}
$$

It is known that there exists at least a common Hamilton sequence formed by Strebel differentials for all extremal differentials in $[\mu]$ (see [1, 3]). Suppose $\mu$ is an extremal Beltrami differential in its class $[\mu]$ and $\left\{\phi_{n}\right\}$ is a Hamilton sequence for $\mu$. The following question was posed by $\mathrm{Li}$ in [3].

Problem. Is the sequence $\left\{\phi_{n}\right\}$ always a Hamilton sequence for all extremal differentials in $[\mu]$ ?

The problem is of interest only when $T(S)$ is infinite-dimensional. Up to now, we have an affirmative answer, given by Wu [5], only when $S=\Delta$.

Theorem B. Let $[\mu]$ be in $T(\Delta)$ where $\mu$ is an extremal differential. Then a Hamilton sequence $\left\{\phi_{n}\right\}$ for $\mu$ is a Hamilton sequence for all extremal differentials in $[\mu]$.

The aim of this paper is to show that the answer is also positive when $S$ is a doubly-connected domain. Up to conformal mappings, we may assume that $S$ is either $\Delta^{*}=\Delta \backslash\{0\}$ or a ring domain $U_{r}=\{z \in \mathbb{C}: 1<|z|<r\}$ for some $r>1$.

Theorem 1.1. Let $S$ be a doubly-connected domain in the complex plane. Suppose that $\mu$ is an extremal Beltrami differential at a point $\tau$ of $T(S)$ and $\left\{\phi_{n}\right\}$ is a Hamilton sequence for $\mu$. Then the sequence $\left\{\phi_{n}\right\}$ is a Hamilton sequence for all extremal differentials in $\tau$. 


\section{Proof of Theorem 1.1}

Let $S=\Delta / \Gamma$ be a doubly-connected domain. Suppose that $f, g \in[f]$ are two extremal quasiconformal mappings from $S$ onto $f(S), g(S)$, respectively. Let $\mu$ and $v$ be the Beltrami differentials of $f$ and $g$, respectively. Let $\widetilde{f}$ and $\widetilde{g}$ be their lifts such that $\left.\widetilde{f}\right|_{\partial \Delta}=\left.\widetilde{g}\right|_{\partial \Delta}$; accordingly, let $\widetilde{\mu}$ and $\widetilde{v}$ be the lifts of $\mu, v$, that is, they are the Beltrami differentials of $\widetilde{f}, \widetilde{g}$, respectively. Since the covering transformation group $\Gamma$ is an Abelian group generated by a conformal self-mapping of $\Delta, \widetilde{f}$ and $\widetilde{g}$ are still extremal in the class $[\tilde{f}]$ by $[4$, Theorem 1$]$.

It is well known that the lift $\widetilde{\mu}$ of $\mu$ satisfies (as does $\widetilde{v}$ ) the $\Gamma$-invariance condition

$$
(\mu \circ \gamma) \overline{\gamma^{\prime}} / \gamma^{\prime}=\mu \text { for all } \gamma \in \Gamma \text {. }
$$

Let $\phi$ be an element of $\mathcal{A}(\Gamma)$ and $\widetilde{\phi}(z) d z^{2}$ be the lift of $\phi$. Then $\widetilde{\phi}$ satisfies

$$
\widetilde{\phi}(\gamma(z))\left[\gamma^{\prime}(z)\right]^{2}=\widetilde{\phi}(z), \quad \gamma \in \Gamma, z \in \Delta .
$$

On the other hand, there exists a holomorphic quadratic differential $\Phi(z) d z^{2} \in \mathcal{A}(1)$ such that the Poincaré series of $\Phi$,

$$
\Theta_{\Gamma} \Phi(z)=\sum_{\gamma \in \Gamma} \Phi(\gamma(z))\left[\gamma^{\prime}(z)\right]^{2},
$$

is equal to $\widetilde{\phi}$ (see $\left[2\right.$, Ch. 4 , Theorem 3]). For every $\phi \in \mathcal{A}_{1}(\Gamma)$, define

$$
I(\phi)=\inf \left\{\|\Phi\|_{\Delta}: \Theta_{\Gamma} \Phi=\widetilde{\phi}, \Phi \in \mathcal{A}(1)\right\} .
$$

Since $\Gamma$ is also an infinite cyclic group, [4, Lemma 3] tells us that $I(\phi) \equiv 1$ for all $\phi \in \mathcal{A}_{1}(\Gamma)$.

Now, assuming that $\left\{\phi_{n}: \phi_{n} \in \mathcal{A}_{1}(\Gamma)\right\}$ is a Hamilton sequence for $\mu$, we need to prove that

$$
\lim _{n \rightarrow \infty}\left|\iint_{S} v(z) \phi_{n}(z) d x d y\right|=\|v\|_{\infty}=k_{0}([\mu]) .
$$

Let $\Omega$ be a fundamental region for $\Gamma$ in $\Delta$. Let $\widetilde{\phi}_{n} d z^{2}$ be the lift of $\phi_{n}$. Then

$$
\iint_{S} \mu(z) \phi_{n}(z) d x d y=\iint_{\Omega} \widetilde{\mu}(z) \widetilde{\phi}_{n}(z) d x d y .
$$

Since $I\left(\phi_{n}\right) \equiv 1$, we can choose $\Phi_{n}(z) d z^{2} \in \mathcal{A}(1)$ such that $\Theta_{\Gamma} \Phi_{n}=\widetilde{\phi}_{n}$ and

$$
\left\|\Phi_{n}\right\|_{\Delta}=1+o\left(\frac{1}{n}\right) \quad \text { as } n \rightarrow \infty .
$$

We easily derive

$$
\begin{aligned}
\iint_{\Omega} \widetilde{\mu}(z) \widetilde{\phi}_{n}(z) d x d y & =\sum_{\gamma \in \Gamma} \iint_{\Omega} \widetilde{\mu}(z) \Phi_{n}(\gamma(z))\left[\gamma^{\prime}(z)\right]^{2} d x d y \\
& =\sum_{\gamma \in \Gamma} \iint_{\gamma(\Omega)} \widetilde{\mu}(z) \Phi_{n}(z) d x d y=\iint_{\Delta} \widetilde{\mu}(z) \Phi_{n}(z) d x d y .
\end{aligned}
$$


Thus, combining (2.1)-(2.3),

$$
\begin{aligned}
\|\mu\|_{\infty} & =\lim _{n \rightarrow \infty} \iint_{S} \mu(z) \phi_{n}(z) d x d y=\lim _{n \rightarrow \infty} \iint_{\Delta} \widetilde{\mu}(z) \Phi_{n}(z) d x d y \\
& =\lim _{n \rightarrow \infty} \iint_{\Delta} \widetilde{\mu}(z) \frac{\Phi_{n}(z)}{\left\|\Phi_{n}\right\|_{\Delta}} d x d y,
\end{aligned}
$$

which indicates that $\left\{\Phi_{n}(z) /\left\|\Phi_{n}\right\|_{\Delta}\right\}$ is a Hamilton sequence for $\widetilde{\mu}$. Furthermore, by Theorem B, it is also a Hamilton sequence for $\widetilde{v}$. Therefore, by the same reasoning as in deriving (2.3),

$$
\begin{aligned}
\|v\|_{\infty} & =\lim _{n \rightarrow \infty} \iint_{\Delta} \widetilde{v}(z) \frac{\Phi_{n}(z)}{\left\|\Phi_{n}\right\|_{\Delta}} d x d y=\lim _{n \rightarrow \infty} \iint_{\Delta} \widetilde{v}(z) \Phi_{n}(z) d x d y \\
& =\lim _{n \rightarrow \infty} \sum_{\gamma \in \Gamma} \iint_{\gamma(\Omega)} \widetilde{v}(z) \Phi_{n}(z) d x d y=\lim _{n \rightarrow \infty} \sum_{\gamma \in \Gamma} \iint_{\Omega} \widetilde{v}(z) \Phi_{n}(\gamma(z))\left[\gamma^{\prime}(z)\right]^{2} d x d y \\
& =\lim _{n \rightarrow \infty} \iint_{\Omega} \widetilde{v}(z) \widetilde{\phi}_{n}(z) d x d y=\lim _{n \rightarrow \infty} \iint_{S} v(z) \phi_{n}(z) d x d y,
\end{aligned}
$$

that is, $\left\{\phi_{n}\right\}$ is also a Hamilton sequence for $v$. This completes the proof of Theorem 1.1.

\section{References}

[1] F. P. Gardiner, 'Approximation of infinite dimensional Teichmüller space', Trans. Amer. Math. Soc. 282 (1984), 367-383.

[2] F. P. Gardiner and N. Lakic, Quasiconformal Teichmüller Theory (American Mathematical Society, Providence, RI, 2000).

[3] Z. Li, 'Strebel differentials and Hamilton sequences', Sci. China Ser. A. 44 (2001), 969-979.

[4] H. Ohtake, 'Lifts of extremal quasiconformal mappings of arbitrary Riemann surfaces', J. Math. Kyoto Univ. 2 (1982), 191-200.

[5] S. Wu, 'Hamilton sequences for extremal quasiconformal mappings of the unit disk', Sci. China Ser. A. 42 (1999), 1033-1042.

GUOWU YAO, Department of Mathematical Sciences, Tsinghua University, Beijing, 100084, PR China

e-mail: gwyao@math.tsinghua.edu.cn 